Editor's Note: These short reviews of a recent paper in the Journal, written exclusively by graduate students or postdoctoral fellows, are intended to mimic the journal clubs that exist in your own departments or institutions. For more information on the format and purpose of the Journal Club, please see http://www.jneurosci.org/misc/ifa_features.shtml.

\title{
Swimming with and against the Stream: Does Motor Adaptation to Lateral Forces Influence Visual Motion Perception?
}

\author{
Gerrit W. Maus \\ Psychology Department, University of Sussex, Brighton BN1 9QH, United Kingdom \\ Review of Brown et al. (http://www.jneurosci.org/cgi/content/full/27/37/9975)
}

Many studies have investigated how the visual system influences motor control (Jackson and Husain, 1997). The reverse relationship of how the motor system influences visual perception has been less studied. For example, it has been suggested that when perceiving the actions of others, the observer's motor system provides an internal model that is used for ongoing perceptual predictions (Wilson and Knoblich, 2005). To what extent can information acquired by the motor system be used in visual processing, and in particular for predictions of moving stimuli?

In a recent study in the Journal of $\mathrm{Neu}$ roscience, Brown et al. (2007) show that learning to compensate for a constantly applied lateral force to the hand influences the accuracy of interception movements to a visually presented moving target. While holding a robotic manipulandum with their right hand, participants first underwent a learning phase in which they made short, quick reaching movements to a visually presented target. During learning three inde-

Received Oct. 5, 2007; revised Nov. 3, 2007; accepted Nov. 5, 2007. I thank Beena Khurana, Jim Parkinson, and Ruth Habibi for fruitful discussions and comments on a previous version of this manuscript.

Correspondence should be addressed to Gerrit W. Maus, Psychology Department, University of Sussex, Falmer, Brighton BN1 90H, UK. E-mail: G.W.Maus@sussex.ac.uk.

DOI:10.1523/JNEUROSCI.4545-07.2007

Copyright $\odot 2007$ Society for Neuroscience $\quad$ 0270-6474/07/2713367-02\$15.00/0 pendent groups of participants were exposed to a force of $10 \mathrm{~N}$, pushing their hand either left or right, or to no force (the control group). Participants quickly learned to compensate for the external force. For those participants under the influence of either force direction, hand movement deviations from a straight line to the target quickly approached equivalent values to those in the control group [Brown et al. (2007), their Fig. 2 (http:// www.jneurosci.org/cgi/content/full/27/ 37/9975/F2)]. In the next phase, participants performed an interception task. With the same force as in the training phase applied to their hand, participants now had to "stab" a rightward moving accelerating target "fish" [Brown et al. (2007), their Fig. $1 B, D$, (http://www. jneurosci.org/cgi/content/full/27/37/ 9975/F1)]. The target accelerated at 10 $\mathrm{m} / \mathrm{s}^{2}$, with motion starting at different initial speeds to achieve different viewing times (472, 560, and $672 \mathrm{~ms}$ ) of the target.

Brown et al. (2007) analyzed both the interception success rate and the "interception time difference," the time between when the center of the target and the hand arrived at the interception point. Interception was generally inaccurate ( $40 \%$ successful hits in the control group), but significantly better ( $\sim 50 \%$ hits) in the group exposed to a force matching the motion of the target (rightward), and lowest ( $\sim 35 \%$ hits) in the group exposed to a force opposite to the direction of target motion [Brown et al. (2007), their Fig. 3 (http://www.jneurosci.org/cgi/content/ full/27/37/9975/F3)]. On average, participants reached the interception point after the center of the target. However, this lag was significantly shorter in the rightward force group than in both the leftward force and control groups. When separately analyzing trials with different viewing times, an effect of force conditions on interception time differences was only apparent for the longest viewing time [Brown et al. (2007), their Fig. 3 (http:// www.jneurosci.org/cgi/content/full/27/ 37/9975/F3)], leading Brown et al. (2007) to argue that the information acquired by the motor system influences interception performance only when participants use a predictive, rather than reactive strategy.

The actual kinematics of the interception movement did not differ between the leftward force and rightward force groups, suggesting that the effects of force direction on interception timing were not the result of actual differences in the movement profile of the hand. To further address this point, new groups of participants underwent the same motor learning phase but subsequently performed a "remote interception" task. Again their right hand was exposed to the same force as during learning, but instead of moving their hand to intercept the target, they pressed a button with their left hand when 
they estimated the target to be in front of their right hand. Similar results were observed, with force direction that is congruent with target motion significantly improving interception timing, but not interception success rates [Brown et al. (2007), their Fig. 5 (http://www.jneurosci. org/cgi/content/full/27/37/9975/F5)].

Brown et al. (2007) propose that motor learning of the kinematic effects of external forces on the arm gives rise to a predictive model that is then shared by the visual system in predicting target motion. To rule out mere multisensory integration or explicit cognitive effects, the authors conducted two additional experiments. The learning phase was eliminated and it was carefully assured that participants did not have any opportunity to move their hand under the presence of an external force. Instead, the force was only applied when participants held their right hand still. Again, a button press of the left hand indicated an interception response to the moving target. To avoid adaptation to the force, the direction alternated every five trials in one group and every 10 trials in another. Neither group exhibited a difference in performance or interception tim- ing between the two force directions, although participants were able to judge directions accurately. These experiments show that motor learning is necessary for the effect. However, whether it is sufficient remains to be examined. In all conditions, participants were able to explicitly identify force direction, whereas in the last two experiments the elimination of motor learning was confounded with a lack of knowledge about the spatial extent of the force field. Therefore a contribution of high-level cognitive processes cannot be ruled out completely.

In summary, Brown et al. (2007) provide an example of how performance in a visual interception task can be influenced by information acquired by the motor system. They demonstrate that target motion is better predicted under the presence of an environmental force that is congruent with target motion, but only after sufficient visual exposure and motor experience to compensate for the force. The effect is most robust when the interception response is an actual movement under influence of the external force. However, as the authors acknowledge, the extent to which motor experience does influence the actual perception of the visual target, remains an open question. Combining their paradigm with motion direction discrimination tasks (Wohlschläger, 2000), or visual localization tasks for either moving objects or static flashes (Nijhawan and Kirschfeld, 2003), might show whether information acquired by the motor system does indeed affect visual motion perception per se, or if it more generally can bias visual, or multisensory, space as a whole.

\section{References}

Brown LE, Wilson ET, Goodale MA, Gribble PL (2007) Motor force field learning influences visual processing of target motion. J Neurosci 27:9975-9983.

Jackson SR, Husain M (1997) Visual control of hand action. Trends Cogn Sci 1:310-317.

Nijhawan R, Kirschfeld K (2003) Analogous mechanisms compensate for neural delays in the sensory and the motor pathways: evidence from motor flash-lag. Curr Biol 13:749-753.

Wilson M, Knoblich G (2005) The case for motor involvement in perceiving conspecifics. Psychol Bull 131:460-473.

Wohlschläger A (2000) Visual motion priming by invisible actions. Vision Res 40:925-930. 